DAVID LUBARSKY, MD, MBA

Emanuel M. Papper Professor and Chair,

Department of Anesthesiology, Perioperative Medicine, and Pain Management,

and Senior Associate Dean for Safety, Quality, and Risk Prevention,

University of Miami Miller School of Medicine, Miami, FL

\section{KEITH CANDIOTTI, MD}

Associate Professor of Anesthesiology and Internal Medicine,

and Chief, Division of Perioperative Medicine,

Department of Anesthesiology, Perioperative Medicine, and Pain Management,

University of Miami Miller School of Medicine, Miami, FL

\title{
Giving anesthesiologists what they want: How to write a useful preoperative consult
}

\section{ABSTRACT}

Anesthesiologists are the primary users of preoperative medical consultations (consults), but the information in consults is often of limited usefulness to anesthesiologists and the rest of the surgical and perioperative team. The purpose of a consult is not to "clear" a patient for surgery but rather to optimize a patient's underlying disease states before they are compounded by the insult of surgery. Too often consults provide advice on subjects that are in the realm of expertise of the anesthesiologist—such as the type of anesthesia to administer or what intraoperative monitoring to use - and thus risk being ignored. Consults should instead provide specific data about the patient that are pertinent to the surgery, as well as guidance on preoperative and postoperative disease management.

\section{KEY POINTS}

Consults that provide pertinent quantitative data about the patient are helpful-eg, the heart rate at which ischemia was exhibited during stress testing and the degree of ischemia.

Anesthesiologists do not need assistance with managing intravenous drugs (with the exception of unusual agents), but they can use specific guidance on managing oral medications pre- and postoperatively to best achieve optimization and steady-state concentrations.

Pertinent recent information ( $<5$ years old) from the nonanesthesiology literature should be provided.

Medical consultants should arrange for follow-up care for patients with active conditions not addressed by the surgery.

Absolute recommendations should be avoided in a consult: the surgical team may have good reason not to follow them, and legal repercussions could ensue. The words "consider" or "strongly consider" usually suffice, except where there is an absolute standard of care.
T

he ideal preoperative medical consultation (consult) is useful to the whole surgical team, ensures maximal patient readiness for surgery, and promotes optimal perioperative care of the patient. Too often, however, consults are ignored or, even worse, set the stage for legal problems. This article identifies problems frequently seen in preoperative consults, particularly from the perspective of anesthesiologists, and gives guidance to those who write consults-hospitalists, internists, cardiologists, and other medical consultants —on providing the information that is most needed by those who use them.

\section{A WIDE RANGE OF END USERS}

Anesthesiologists are most often the primary users of the information in preoperative consults, but many other members of the surgical and perioperative team benefit from a well-developed consult, including surgeons, intensivists, nurses, and pain management specialists. Most important, patients stand to benefit, as a good consult helps to ensure that the full breadth of relevant patient-specific information is brought to bear to anticipate potential difficulties and promote optimal care.

\section{Purpose of a consult is in the eye of the beholder}

The literature on medical consults in the perioperative arena is scant. The only fairly recent assessment of physician attitudes toward the role of consults was reported by Katz et al in 1998. ${ }^{1}$ These researchers surveyed attitudes about the various perceived purposes of preoperative cardiology consults, and received rather different responses from anesthesiologists, cardiologists, and surgeons.

There was consensus among all three specialties that two particular functions of a consult are important:

- Treating an inadequately managed cardiac condition before surgery

- Providing data to use in anesthetic management.

Additionally, all three specialties deemed the suggestion of intraoperative treatment modalities to be reasonably important when such suggestions were specifically included in the consult request, although anesthesiologists assigned less importance to this function. ${ }^{1}$

In contrast, anesthesiologists considered suggestions about intraoperative treatment generally unimportant when not specifically requested, and they viewed suggestions of intraoperative monitoring and advice on the 
safest type of anesthesia as even less important. Anesthesiologists also deemed "clearing the patient for surgery" as an unimportant function of the consult. Cardiologists rated all of these functions as more important than anesthesiologists did and in some cases as considerably more important. To many of the survey questions, surgeons responded that a specific purpose of a consult was "neither important nor unimportant." "That may be because the surgeon's purpose in obtaining the consult is often simply to address the concerns of the anesthesiologist, who might otherwise delay or cancel a needed surgery.

\section{Consult deficiencies:} Vagueness, illegibility, dictating anesthetic choice The survey by Katz et al also assessed each specialty's perceptions of the most common deficiencies of preoperative cardiology consults. The deficiencies deemed most common were failure to give specific facts, illegible handwriting, and attempts to dictate the type of anesthesia to be used. Anesthesiologists considered each of the deficiencies assessed as occurring more commonly than their cardiologist or surgeon colleagues did. ${ }^{1}$

\section{The requester-user disconnect}

The differing perceptions of preoperative consults by anesthesiologists and surgeons underscore a fundamental problem: the primary requesters of consults (surgeons) are different from the primary users of consults (anesthesiologists).

Ideally, preoperative consults should be requested by anesthesiologists. Unless and until the ordering of consults changes on a wide scale, however, our advice is for consultants to ask the anesthesiologist what he or she needs to know, in addition to any questions directed to the requesting surgeon. Communication between the surgeon and anesthesiologist should be encouraged as much as possible, and consultants should keep both the anesthesiologist and surgeon in mind when writing consult notes.

\section{A final end user: The plaintiff's attorney}

It is wise to keep in mind one more potential user of your consult: a plaintiff's attorney. A poorly written consult may benefit plaintiffs' lawyers. Consults should never give absolute instructions; it is better to use such phrases as "Strongly consider..." or "The current literature strongly suggests..." Otherwise, the surgical team is placed in an awkward position if it does not follow your recommendations, even if for good reason. If a certain recommendation absolutely must be followed, then direct oral communication from the consultant to the attending anesthesiologist (or surgeon) is best.

\section{CONSIDER THE PRIMARY USER: WHAT ANESTHESIOLOGISTS ALREADY KNOW}

For the purpose of preoperative consults, it is helpful to think of anesthesiologists as experts in acute medical care. Their 4-year training consists of the following:
- 1 year of internship, often in medicine, including 6 months of basic patient care in the ward or clinic (the last time they will manage chronic disease)

- 4 months in the intensive care unit (ICU) and 1 month in the recovery room, which yields solid intensivist training

- 3 months in pain management, covering acute and chronic pain and regional blocks

- $\sim 24$ months in the operating room, often devoted to care of complex problems in surgical subspecialties (obstetric, pediatric, neurologic, cardiothoracic, vascular)

- 1 month of preoperative screening and consultations (a recent requirement).

An optional fifth year may be spent in a subspecialty.

Since the large part of anesthesiologists' training is in acute care, they generally do not need advice about the acute treatment of any ailment. Consults should not advise anesthesiologists on subjects in which they have considerable expertise. They already have well-established ideas about addressing hypertension, myocardial ischemia, heart failure, arrhythmias (unless unusual therapies are needed), bronchospasm, glucose levels, and pain in the operating room, so they are apt to ignore advice on such topics.

There are several additional topics in which anesthesiologists have considerable expertise and do not need guidance in consults:

- Choice of anesthetic type and its impact on outcome

- Choice of invasive or noninvasive monitoring for any comorbidity and operation

- Postoperative patient disposition (ie, whether to send a patient home, to the postanesthesia care unit, to the ICU, or to a step-down unit)

- Impact of optimizing organ function on perioperative outcome

- Cardiovascular and respiratory physiology

- Pharmacology of intravenous agents.

\section{WHAT ANESTHESIOLOGISTS MAY NOT KNOW- AND NEED FROM CONSULTANTS}

\section{How to manage chronic diseases}

Preoperative consults should concentrate on matters in which anesthesiologists are not well trained (Table 1). These largely involve optimizing the preoperative treatment of chronic diseases-eg, hypertension, diabetes, coronary artery disease, renal failure, malnutrition, hepatic dysfunction, asthma/chronic obstructive pulmonary disease-and managing oral drug regimens. Anesthesiologists generally do not need help, however, in optimizing the function of an organ system once the patient is in the operating room. Advice on preoperative optimization should include guidance on how long the optimization is likely to take.

Follow-up care (eg, for poorly controlled diabetes or hypertension) often can wait until after the operation, and a consultant's opinion about that is appreciated. It is especially 
TABLE 1

Useful information to include in preoperative consults*

- How to preoperatively optimize function of an unhealthy organ system

- Guidance on managing oral drug regimens

-First-line and second-line agents

-Initial dosage and titration; recommended combinations

-How to manage side effects

- Expected time until patient is optimized for the procedure if above management is followed

- Tests that might be indicated preoperatively to direct therapy to optimize function

- Additional interventions indicated by the patient's disease, and appropriate timing (pre-, intra-, postoperatively)

-Include assurance that consultant will follow up with specified nonurgent postoperative care without prompting

- Current pertinent anticoagulation recommendations

- Details on coronary stents-when placed, where placed, and type (drug-eluting or bare metal)

- Focused information on cardiac defibrillators and other implanted devices, specifically:

-Whether patient is pacer-dependent

-Effect of magnet placement

- Has battery recently been checked?

- Recommendations on intra-/perioperative management of:

-Rare diseases

-Blood disorders, especially coagulation abnormalities

-Brittle diabetes (loading doses, optimal make-up of infusions, treatment targets)

-Endocrine disorders (eg, perioperative dosing of thyroid drugs)

- Newer recommendations/data ( $<5$ years old) on acute medical management, especially in patients with complex comorbidities

- Explanations/references when recommendations deviate from accepted guidelines

- Legible contact information, including an emergency phone number to ensure access prior to early-morning procedures

* In all cases, be as specific as possible and favor quantitative over qualitative information when possible.

helpful to know that the patient will be followed without the surgeon or anesthesiologist having to arrange for it.

\section{New evidence-based guidance from the literature}

One case when recommendations on acute medical management should be provided is when they involve new information from the literature-ie, important data or guidelines published within the prior 5 years or so. It can take time for new information and recommendations to reach all clinicians even within a single specialty. Moreover, important information, such as on the perioperative use of beta-blockers and statins, is not necessarily published in the anesthesiology literature. It is critical to relay information such as the recent recommendation not to withdraw statins prior to surgery, as the current editions of most anesthesiology textbooks have incorrect information suggesting discontinuation. Thus, consultants should include pertinent recent data and guideline recommendations, especially if they go against previous dogma.

\section{Rare diseases, blood disorders, other special cases}

As outlined in Table 1, advice on perioperative management is appreciated for patients with rare diseases, coagulation disorders or other blood disorders, and brittle diabetes and other endocrine disorders, as most anesthesiologists are not intimately familiar with these conditions. Anesthesiologists also need, but often do not get, basic details on coronary stents and other implanted devices (see Table 1), as well as guidance on the latest anticoagulation recommendations, with which it is difficult to keep up to date.

\section{A sensitivity to audience and context}

It is always appropriate to ask the surgeon requesting a consult-and the anesthesiologist assigned to the case, if known - what he or she wants to know from the consult. If guidance on specific cases is impractical, it is appropriate to ask the chair of the anesthesiology department, or several anesthesiologists collectively, for general guidance on what they look for from preoperative consults.

Anesthesiologists, like consultants, comprise a broad range of people, and it is always important to be sensitive to context. Generalists who work mainly on healthy patients or in a community setting may have forgotten some of their training in acute medicine and are more likely to appreciate advice on intraoperative care. On the other hand, an anesthesiologist who trained in a cardiothoracic subspecialty fellowship, who routinely deals with issues such as left ventricular assist devices and heart transplants, would not benefit from such advice.

\section{WHAT A CONSULT SHOULD—AND SHOULD NOT—BE}

The above advice can be distilled into a few principles:

- A consult is an opportunity for the medical consultant to provide helpful management suggestions to the operative team.

- A fundamental objective of a consult is to optimize a patient's underlying disease before it is compounded by the insult of surgery.

- The purpose of a consult is never to "clear" a patient for surgery. Whether or not to proceed to surgery is a question for the anesthesiologist, surgeon, and patient to decide after weighing the risks and benefits once the patient's comorbidities are optimally managed. The consult is an important contributing factor to this decision, but it should never be the mechanism of the decision. Though the note from the surgeon requesting a consult may routinely be written as, "Clear the patient for surgery," consultants should recognize this for what it is - the surgeon's attempt to avoid having the anesthesiologist cancel the operation-and refrain from weighing in on "clearance" one way or the other. 


\section{CASE STUDY: CARDIAC CONSULT REQUESTED BEFORE FEM-POP BYPASS SURGERY}

Cardiovascular problems are the most common reasons for requesting preoperative consults. The following case illustrates a typical scenario for a cardiac consult request and presents examples of good and bad notes requesting consults and good and bad consults written in response.

\section{The case}

A 47-year-old man is scheduled for femoral-popliteal bypass surgery. His medical history is significant for diabetes, a myocardial infarction (MI) 3 years ago followed by placement of a stent, and a limited ability to assess exercise tolerance. Evidence of an anteroseptal MI is present on 12-lead electrocardiography. His medications include metoprolol $25 \mathrm{mg}$ twice daily and an oral hypoglycemic agent. His blood pressure is $152 / 89 \mathrm{~mm} \mathrm{Hg}$, heart rate 81 beats per minute, respiratory rate 14 breaths per minute, and arterial oxygen saturation $96 \%$ on room air.

\section{The consult request: Bad and good examples}

A bad consult request in this case would be, "Clear the patient for surgery." Although this type of request is routinely written, it is routinely useless.

For this complex surgery with significant fluid requirements, a much better consult request would include several specific requests and questions and might read as follows:

-Please evaluate patient's post-MI therapy for his CAD. Is further therapy required to optimize CAD treatment?

-Do his blood pressure or diabetic regimens need modification? If so, can this be done postoperatively?

-Please evaluate patient's myocardial function in light of a lack of info on exercise tolerance. Is an echo indicated?

-Are other tests, therapies, or interventions warranted pre-or postoperatively?

\section{Example of a bad consult}

A poorly written consult in a case like this may:

- Include a brief history repeating facts that are already known and noting that "the patient is at his baseline without obvious ischemia."

- State that the patient is cleared for "spinal" anesthesia. "Clearing a patient for anesthesia" is useless to begin with, but clearing for a certain type of anesthesia places the anesthesiologist in a terrible medicolegal position if general anesthesia turns out to be needed. Moreover, there are no proven major outcome differences related to the type of anesthetic chosen.

- Recommend that "a pulmonary artery catheter might be indicated to monitor hemodynamics." Besides the fact that such catheters probably do more harm than good, such a recommendation is unnecessary since the anesthesiologist is already expert in managing perioperative care.

- Recommend that "the anesthesia team should monitor the patient carefully in the perioperative arena for hypoxia and hypotension." Qualitative advice, such as "avoid hypoxia, hypotension, and tachycardia," is not useful, but quantitative information, such as "during ischemic stress testing, the patient exhibited ischemia when his heart rate went to 142 ," can be very helpful.

- State that the patient be sent to the ICU following surgery. Mandating an ICU stay in advance makes no sense unless the operation itself demands ICU care, which is the call of the surgeon and anesthesiologist anyway.

A consult like this doesn't tell the perioperative team anything that it didn't already know. Such a consult not only is unhelpful but also actually creates more work since much of the advice needs to be "undone" lest a lawyer see the chart and it not be clear why the consultant's recommendations were not followed.

\section{Example of a good consult}

In contrast, a good consult for this case would involve:

- A detailed history examining the potential for silent ischemia associated with the diabetes, as well as the relationship of the hypertension and beta-blocker therapy to episodes of ischemia. The level of ischemia should be clearly categorized. If it cannot be determined, this should be noted; if it can be determined only that the ischemia is not New York Heart Association class III or IV, note this as well (the perioperative outcomes literature suggests that no preoperative ischemia testing is needed with class I or II angina).

- Guidance on blood pressure optimization in light of the relative urgency of the procedure. Blood pressure need not be normalized preoperatively in this case, but if the operation were totally elective and the consultant felt it could make a difference, it would be appropriate to suggest that blood pressure be optimized beforehand.

- A recommendation on whether and when to change the beta-blocker dosage. If the dosage needs to be increased, the anesthesiologist will want to know how many doses are needed to reach a new steady state. Joint guidelines from the American College of Cardiology (ACC) and American Heart Association (AHA) ${ }^{2}$ recommend 7 to 30 days, but this time frame is unrealistic in this setting, so more practical advice would be appreciated. A good consult notes any deviation from established guidelines, however, and explains the rationale for such deviation.

- Evaluation of the myocardium at risk. This is especially important with left main disease, as it influences the decision whether to test or intervene versus proceeding with careful beta-blocker titration.

- Evaluation of myocardial function and, if appropriate, a therapy suggestion for optimization.

- Notation of the heart rate or blood pressure thresholds at which ischemia develops if a stress test is performed.

\section{GOOD GUIDANCE FROM THE ACC/AHA GUIDELINES}

Our advice here is broadly consistent with the aforementioned 2007 ACC/AHA guidelines on perioperative 
cardiovascular evaluation for noncardiac surgery. ${ }^{2}$ The following observation on cardiac evaluations from these guidelines applies to preoperative consults in general:

The purpose of preoperative evaluation is not to give medical clearance but rather to perform an evaluation of the patient's current medical status; make recommendations concerning the evaluation, management, and risk of cardiac problems over the entire perioperative period; and provide a clinical risk profile that [can be used] in making treatment decisions that may influence short- and long-term cardiac outcomes. ${ }^{2}$

These guidelines contain a good description of the ideal preoperative evaluation and consult in a short section (Section II, "General Approach to the Patient")2 that is worthy of wide dissemination.

\section{DISCUSSION}

Question from the audience: Many consults are written more for the surgical team than for the anesthesiologists, hence advice such as managing intraoperative diabetes. Isn't that appropriate?

Dr. Lubarsky: There are a variety of users of the information in a consult note. I focused on the anesthesiologist, but certainly the surgical staff and house staff would benefit from suggestions about postoperative management. However, they would not benefit from suggestions on intraoperative management; surgeons simply do not need this information and the anesthesiologist will have his or her own regimen. But if there is a specific type of insulin infusion that's been shown to be best in the specific patient at hand, then detailing that obviously is beneficial.

Question from the audience: We all agree that communication is key, but how does the consultant reach the anesthesiologist to find out what he or she wants to know when the anesthesiologist isn't usually assigned to the case until a day before surgery?

Dr. Lubarsky: If no anesthesiologist is yet assigned to a case, the consultant can discuss the case with the chief of the anesthesiology department. The discussion should be documented in the note. But it's important that the system be changed so that anesthesiologists are assigned to cases well in advance. I instituted such a policy at my previous hospital. Many hospitals schedule surgeries 3 months in advance, and many anesthesiology departments have schedules made at least 1 month and often 2 to 3 months in advance. The department could assign a specific anesthesiologist to a future scheduled case with ease.

Question from the audience: How do anesthesiologists educate all the various people we rely on for consults when we can't get them in one place at one time?

Dr. Lubarsky: It's a challenge. I try many things, such as going to cardiology rounds, but there are always new people coming through. A good monograph or a set of guidelines with examples would help. If each specialty educates the other and speaks at each other's conferences more often, that should help. Anesthesiologists would benefit from hearing about the challenges medical consultants face; we may not be doing all we can to optimize perioperative care. There's room for improvement through communication on both sides. I should also emphasize that we're all trying to do the right thing. Doctors try to be accommodating, but that doesn't always make for good decisions. Recently a consultant in my hospital did a preoperative stress test on a patient who didn't need one. When I asked why, he said, "Because the surgeon asked me to."

Question from the audience: But don't you agree that many anesthesiologists would like to see that negative stress test, even if a stress test is not indicated by the guidelines? Cardiologists know that the anesthesiologists are often looking for that on the morning of surgery.

Dr. Lubarsky: The point is that physicians should be responsible for what they have expertise in. When I am asked to intubate a patient, my response as an expert in intubation might be, "Actually, he doesn't need to be intubated right now." In the case of this unnecessary stress test, the cardiologist probably should have called the surgeon and said, "It's really not indicated because the patient had a negative stress test 2 years ago, there's been no change in symptoms and no angina since then, and he operates well above 4 metabolic equivalents. There's a clear-cut reason not to do it." If the surgeon still wanted the test done just to be reassured, that's simply a poor use of society's resources. We depend on experts to identify the tests that are indicated to evaluate a patient's disease and not just do tests for the sake of doing them.

\section{DISCLOSURES}

Dr. Lubarsky has indicated that he has relevant financial relationships with the following commercial interests: advisory board of MGI Pharma; consultant to Abbott Laboratories; grants/research support from Grant Downing; salary/ contractual services for Abbott Laboratories; and speakers' bureaus of Schering-Plough and Pfizer. Dr. Candiotti has indicated that he has no financial relationships with commercial interests that have a direct bearing on the subject matter of this article. All conflicts of interest have been resolved.

This article was developed from an audio transcript of Dr. Lubarsky's lecture at the 4th Annual Perioperative Medicine Summit. The transcript was edited by the Cleveland Clinic Journal of Medicine staff for clarity and conciseness, and was then reviewed, revised, and approved by Drs. Lubarsky and Candiotti.

\section{REFERENCES}

1. Katz RI, Barnhart JM, Ho G, et al. A survey on the intended purposes and perceived utility of preoperative cardiology consultations. Anesth Analg 1998; 87:830-836.

2. Fleisher LA, Beckman JA, Brown KA, et al. ACC/AHA 2007 guidelines on perioperative cardiovascular evaluation and care for noncardiac surgery: a report of the American College of Cardiology/American Heart Association Task Force on Practice Guidelines. Circulation 2007; 116:e418-e499.

Correspondence: David A. Lubarsky, MD, MBA, Department of Anesthesiology, University of Miami Miller School of Medicine, P.O. Box 016370 (R-370), Miami, FL 33101-6370; dlubarsky@med.miami.edu 